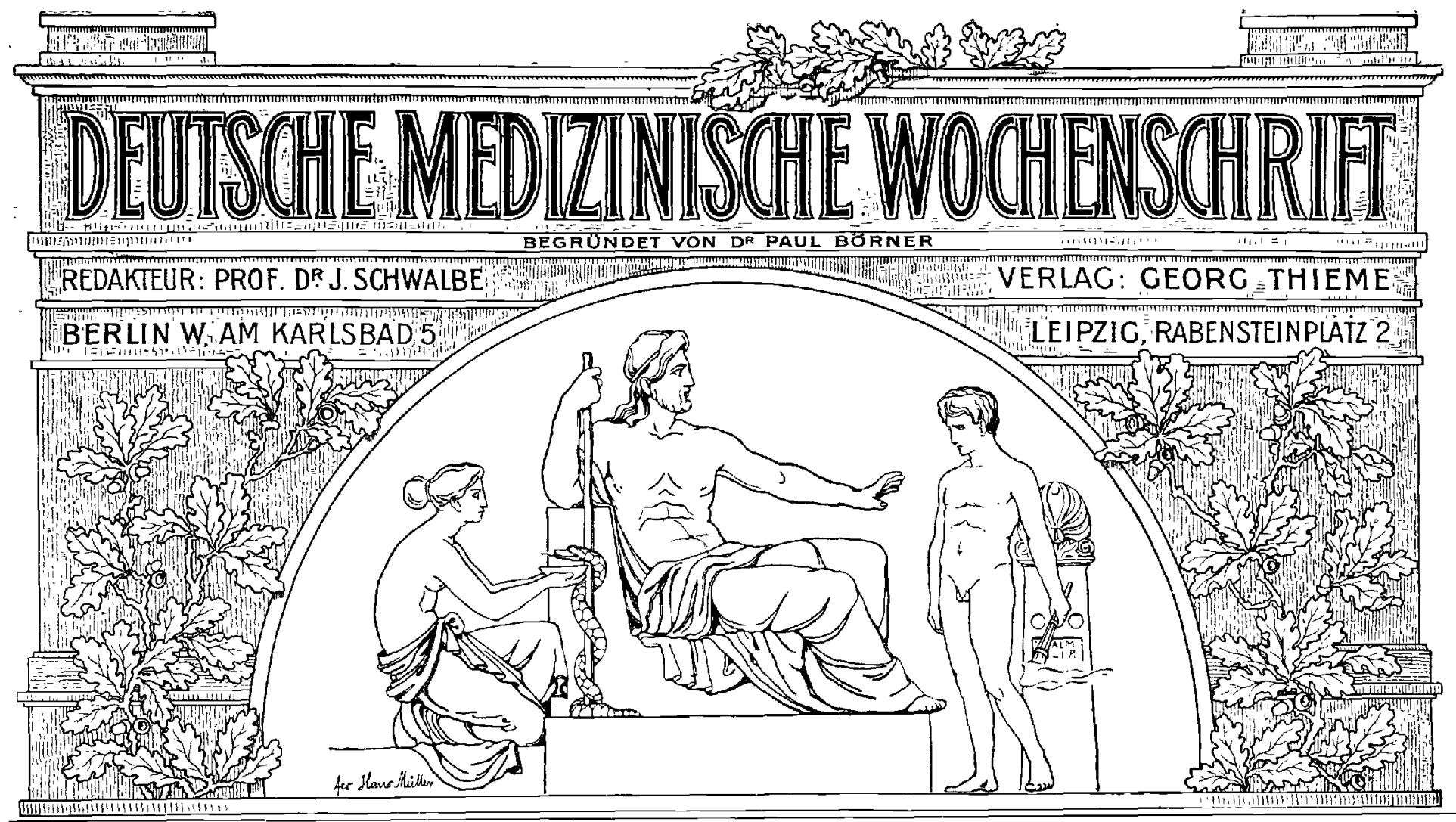

No. 11.

Donnerstag, den 15. März 1906.

32. Jahrgang.

\section{Zur Frühdiagnose und Behandlung des Kehlkopfkrebses.}

Fortbildungsvortrag von Prof. Dr. B. Baginsky in Berlin.

Die ausgedehnten Forschungen der letzten Jahre über die Entstehung und das Wesen der Carcinome haben die durch die bisherigen Beobachtungen allgemein angenommene und gewährleistete Tatsache, daß das Carcinom in seinem Beginn und oft noch sehr lange ein örtliches Leiden ist, nur bestätigen können, und deshalb hat auch die ärztliche Kunst seit langer Zeit es schon als die vornehmste Aufgabe betrachtet, den lokalen Herd so schnell als möglich aus dem Körper zu eliminieren, will man nicht Gefahr laufen, der Infektion Tor und Tür zu öffnen. Dieses Bestreben setzt naturgemäb die frühzeitige Erkenntnis des Leidens voraus, ohne welche selbstverständlich ein zielbewußtes Handeln ausgeschlossen ist. Je kleiner der zu entfernende Tumor ist, je weniger er Gelegenheit hat, in die Nachbarschaft hineinzuwuchern, desto leichter ist die Möglichkeit der ohne grobe Verstïmmelung erreichbaren radikalen Entfernung gegeben, und auch die Gefahr etwaiger Rezidive wird bei der Eigenartigkeit der Verbreitung solcher Affektionen erheblich vermindert.

Dies gilt wie für die Carcinome im allgemeinen in noch erhöhterem Maße für die Larynxcarcinome, berücksichtigen wir die Dignität des befallenen Organs, welches für die Atmung und die Stimmbildung in gleicher Weise in Betracht kommt, und die Gefahren, welche dem Organismus infolge der Nachbarschaft so vieler lebenswichtiger Teile bei etwaigem Uebergreifen des pathologischen Prozesses auf diese drohen.

Der Glaube, dab die malignen Tumoren des Kehlkopfes und speziell die Carcinome $\mathrm{zu}$ den seltenen Affektionen gehören, wurde mit der Einführung des Kehlkopfspiegels in die ärztliche Untersuchungstechnik erschüttert. Indem man in die Lage kam, den pathologischen Veränderungen des Larynx mit dem Spiegel nachgehen zu können, ließen sich als Ursache mannigfacher Störungen Geschwulstbildungen im Kehlkopf erkennen; man lernte sie in ihrem klinischen Verhalten beurteilen und nicht minder durch sorgfältige pathologisch-anatomische Untersuchungen klassifizieren.

Neben einer großen Zahl gutartiger Tumoren, welche nach ihrer operativen Beseitigung keine Tendenz zur Rezidivierung zeigen, lieben sich im Kehlkopf auch bösartige feststellen, Carcinome und Sarkome, welche durch die Art ihrer zerstörenden Eigenschaften sich auszeichnen, dnrch die Gröbe ihres Wachstums und besonders durch die ausgesprochene Neigung der lokalen Rezidivierung und der Metastasenbildung, und gan $z$ besonders sind es die Carcinome, welche die häufiger hier vorkommende Art vorstellt, während die Sarkome relativ seltener sind. Soweit man aus gröberen statistischen Zusammenstellungen ein ungefähres Bild über die Häufigkeit des Vorkommens sich machen kann, ergibt sich hier ein Zahlenverhältnis der gutartigen und bösartigen Tuınoren von etwa 6:1 und bei Carcinomen und Sarkomen ein solches von ungefähr $22: 1$.

Die sonst übliche Unterscheidung der primären und sekundären Carcinome läßt sich auch am Larynx festlıalten, und wir werden zunächst die ersteren, die primär im Larynx auftretenden Carcinome, besprechen und alsdann auch die sekundären behandeln, letztere aber nur insoweit, als es die Fragestellung bezüglich der Frühdiagnose erheischt.

Es kann selbstverständlich nicht meine Absicht sein, einzutreten in die Frage bezüglich der Genese und des Wesens der Carcinome im allgemeinen. Jeder, der sich auch nur cinigermafien angelegen sein läßt, die inedizinische Literatur zu verfolgen, weiB, in welcher Gürung sich alle diese Fragen in diesem Augenblicke befinden, ob lokale Reize für die Aetiologie eine Bedeutung haben etc. und namentlich, wie die parasitäre Lehre, welche von einigen verfochten, von andern dagegen, und mit Recht, verworfen wird, zum Gegenstand ausgiebigster Diskussionen in neuerer Zeit gemacht wird. So interessant alle diese Fragen sind und namentlich auch der Nachweis von Carcinomen bei Kaltblütern (Frl. Plehn, Pick u.a.), so haben die Beobachtungen indes bisher bezüglich der Genese eine Aufklärung irgend welcher Art nicht erbracht, und dasselbe gilt auch für die Larynxcareinome. Sie erscheinen meist in dreifacher Form, und zwar als Epithelialcarcinom; als Faserkrebs und als Medullarcarcinom. Die ersteren konimen am häufigsten vor, sodaß Sendziak in seiner Statistik unter 486 Fällen 248 Epithelialcarcinome fand. In der Mitte steht das Medullarcarcinom, welches noch etwas häufiger als der Faserkrebs zur Beobachtung gelangt Jede Partie des Larynx kann der Ausgangspunkt des Carcinoms sein. obschon nach den Erfahrungen fast aller Kehlkopfärzte eine besondere $\mathrm{Be}$ vorzugung an den wahren Stimmbändern statthat, sodaf Sendziak unter den 486 Fällen 107 an den wahren und 23 an den falschen Stimmbändern feststellen konnte. Seltener kommen Carcinome an den Ventriculi Morgagni und der Plica interarytaenoidea vor, etwas häufiger an der Epiglottis. 
Das makroskopische Aussehen ist wechselnd. Im allgemeinen stellen die Epithelialcarcinome warzige, höckerige Bildungen dar, welche, von verschiedener Größe und Ausdehnung, gegen das normale Gewebe sich absetzen, während das Medullarcarcinom einen äuferst weichen, der Scirrhus einen festen, derben Tumor darstellt. Die Farbe schwankt zwischen gewissen Nüanzierungen von grau und rot, in vielen Fällen bietet sie etwas Weifles, Opakes, namentlich an den Stimmbandcarcinomen, worauf B. Fraenkel zuerst aufmerksam gemacht hat. Bei allen diesen Tumoren besteht die Neigung der allmählichen Größenzunahme, wobei die Ausbreitung im allgemeinen mehr in die Tiefe hinein als an der Oberfläche statthat, obschon auch hier Ausnahmen vorkommen und wiederholt zur Beobachtung gelangt sind. Mit dieser Wachstumszunahme Hand in Hand geht vielfach die Tendenz zum geschwürigen Zerfall, zunächst oberflächlich durch Abschilferung und Exfoliation des Epithels in Form von Erosionen und allmählich mehr in die Tiefe gehend, und es entsteht auf diese Weise ein tiefgehendes Ulcus, indem die zelligen Elemente des Neoplasma zugrunde gehen. Das sind die Bilder, welche wil in den vorgerückten Stadien der Larynxcarcinome bei der Autopsie zu sehen bekommen und in solchen Fällen, in denen die Geschwülste durch Wachstumszunahme eine gewisse Größe erreicht haben und schlieflich im Verlaufe des jauchigen Zerfalls der Tod der Erkrankten eintritt.

Was die dem Kehlkopf benachbarten Lymphdrüsen betrifft. so sehen wir, dab ihre Beteiligung und Mitaffektion beim Kehlkopfkrebs gegenüber anderen Organen eine relativ späte ist, und namentlich trifft dies in vielen Fällen nach den Beobachtungen aller zu für solche Carcinome, welche im Innern des Kehlkopfes gelegen sind und von Krishaber als Cancer intrinsèque bezeichnet worden sind, das sind Carcinome in unmittelbarer Nähe der Glottis, an den wahren und falschen Stirnbändern, an den Ventriculi Morgagni und in der Incisura interarytaenoidea; bei den außerhalb der Kehlkopfhöhle gelegenen Krebsen, dem Cancer extrinséque Krishabers, also am Kehldeckel, der hinteren pharyngealen Kehlkopfwand, den Plicae aryepiglotticae, ist die Mitaffektion der benachbarten Lymphdrüsen im allgemeinen etwas früher, augenscheinlich infolge der reichlicheren Lymphgefäße. welche sich hier vorfinden und die Verbindung mit den Lymphdrüsen vermitteln. Auch spielt die Art des Carcinoms eine wesentliche Rolle mit, indem die harten Krebse, auch wenn sie außerhalb des Kehlkopfes gelegen sind, erst spät Veranlassung zu Lymphdrüsenschwellungen geben.

Die sogenannten sekundären Carcinome des Larynx, welche erheblich seltener als die primären sind, entstehen durch Fortwuchern eines in der Nachbarschaft entstandenen Krebses, und zwar so, dab ein direktes Hineinwachsen in den Larynx erfolgt. Es kann dies von der Zunge, vom Rachen, vom Oesophagus, von der Schilddrüse etc. aus statthaben; selbstverständlich wird je nach dem Sitze des primären Carcinoms sein Eintritt in den Larynx an verschiedenen Stellen leichter oder schwerer erfolgen, wobei auch naturgemäß die Widerstandskraft der Gewebe für das weitere Fortschreiten von großer Bedeutung ist. Auch der Charakter des etwa entstehenden sekundären Larynxcarcinoms ist derselbe wie beim Primärherd, und der Verlauf, resp. der geschwürige Zerfall mit all den bekannten Vorgängen spielt sich in derselben Weise ab wie bei den primären Carcinomen, sodall in späteren Stadien der Ausgangspunkt kaum noch erkannt werden $\cdot$ kann.

Wirklich metastatische Carcinome kommen im Larynx nur äuberst selten vor; es finden sich in dieser Hinsicht nur spärliche Mitteilungen in der Literatur; béobachtet wurde Larynxcarcinom bei allgemeiner Carcinose (Eppinger), bei. Mammacarcinom (M. Schmidt) und einem Falle von Knochencarcinom.

In histologischer Beziehung besteht zwischen den Larynxcarcinomen und denjenigen anderer Organe eine völlige Uebereinstimmung, sodal wir auf eine besondere Darstellung der Strukturverhältnisse Verzicht leisten können

Nachdem wir uns im allgemeinen über die im Kehlkopf rorkommenden Carcinome, soweit die anatomische und patho- logisch-anatomische Untersuchung Aufschluf gibt, orientiert haben, wenden wir uns zur Frage der Diagnose und besonders der Frühdiagnose. Von ihr hängt, wie bereits angegeben, das Wohl und Wehe der Kranken ab; die frühzeitige Erkenntnis des Leidens wird den Arzt zu schnellem Handeln antreiben und ihm die Mittel in die Hand geben, auch frühzeitig die erwünschte und erhoffte Hilfe zu bringen. Soweit es sich um sekundäre Larynxcarcinome oder um das seltene Vorkommnis eines metastatischen Larynxcarcinoms handelt, kann, wie ja selbstverständlich ist, von einer Frühdiagnose kaum die Rede sein, da ja die vorangehenden Krankheitserscheinungen am Primärherd das ganze Krankheitsbild beeinflussen und dementsprechend bei dem Auftreten einer derartigen sekundären laryngealen Neubildung die Diagnose auf Fehlschlüsse kaum stößt, überdies auch die etwaigen Vorteile einer frühen Erkennung immerhin illusorisch werden. Anderseits würden sich unter Berücksichtigung der folgenden Darstellung diejenigen Momente ergeben, welche für die Diagnose auch dieser sekundären und metastatischen Carcinome von Bedeutung sein können. Es kommen also nur die primär im Larynx entstehenden Carcinome in Betracht.

In einer sehr bemerkenswerten Arbeit hat 1889 B. Fraenkel den Frühformen der Kehlkopfcarcinome seine besondere Aufmerksamkeit zugewendet, alle klinischen und diagnostischen Momente ausführlich besprochen und ist zu dem Schlubresultat gekommen, daß der Kehlkopfkrebs in seinem Beginn mit Hilfe des Kehlkopfspiegels und der mikroskopischen Untersuchung herausgenommener Stücke fast ausnahmslos sicher als solcher erkannt werden kann. Die Zahl der seit dieser Zeit zur Beobachtung gelangten laryngealen Carcinome hat sich ganz erheblich gesteigert und mit ihr die Summe der Erfahrungen sowohl mit Bezug auf die Diagnose als auch mit Bezug auf unser operatives Können; nichtsdestoweniger müssen wir, obschon wir B. Fraenkels Ansicht teilen, daß mit Hilfe der Untersuchungsmethoden unter gleichzeitiger Berücksichtigung der begleitenden Umstände der Larynxkrebs in seiner Frühform meist erkannt werden kann, ausdrücklich hervorheben, daß trotzdem diagnostische Irrtümer vorkommen können und auch vorgekommen sind, und daß selbst eine reiche Erfahrung vor Zweifeln nicht schützt. Ich stimme Gottstein bei, welcher gelegentlich der Mitteilung eines Falles von Larynxcarcinom ausdrücklich hervorhebt, dafi es zu den schwierigsten Aufgaben gehört, sich in den früheren Entwicklungsstadien des Carcinoms vor diagnostischen Irrtümern zu schützen, und daß wir oft in der Lage sind, unser Urteil zu suspendieren und den Fall einige Zeit einer sorgfältigen Beobachtung zu unterziehen. Im wesentlichen müssen wir zur Diagnostik heranziehen die genaue, sorgfältige Anamnese, die begleitenden Umstände, das Kehlkopfspiegelbild und schließlich die mikroskopische Untersuchung exstirpierter Stücke, sei es, daß die Exstirpation auf endolaryngealem Wege möglich ist, oder dab sie erfolgt nach vorheriger Spaltung des Kehlkopfes, der Laryngofissur, in solchen Fällen, in denen wegen des Sitzes, der Art der Verbreitung oder aus einem andern Grunde die endolaryngeale probatorische Exstirpation unmöglich erscheint.

Von einer gewissen und nicht zu unterschätzenden Bedeutung sind das Alter und das Geschlecht des Kranken. In jụgendlichen Alter kommen erfahrungsgemäl Carcinome nicht vor, auch ist das weibliche Geschlecht gegenüber dem männlichen darin bevorzugt, daß Frauen auch im späteren Alter relativ selten am Kehlkopfkrebs erkranken. Nach allen vorliegenden Beobachtungen und Statistiken sehen wir, daß bei Männern von 40 Jahren an und darüber die Carcinome am häufigsten vorkommen; nach Morel Mackenzi e wird am meisten betroffen, in $80 \%$ der Fälle, das 40 . bis 70. Lebensjahr. Dementsprechend steigt die Wahrscheinlichkeitsdiagnose mit der Zunahme der Jahre. $O b$ infolge besonderer Schädlichkeiten, des Abusus spirituosorum oder anderer Dinge, die gröBere Disposition auf seiten des männlichen Geschlechtes liegt, ist nicht mit Sicherheit erwiesen; unmöglich ist die Annahme nicht.

Subjektive Symptome, welche für die frühe Diagnose Verwertung finden können, können vorhanden sein, gehören aber nicht direkt zum Krankheitsbilde des eben entstehenden Carcinoms. Es ist klar, dâ, wenn ein derartiges Leiden sich 
primär an den wahren Stimmbändern entwickelt, schon sehr frühzeitig Behinderungen in der Stimmbildung auftreten können, Belegtheit der Stimme, und daß diese Störungen schon früh in Heiserkeit sich äußern können. Anderseits ist aber auch ersichtlich, daß, wenn die Entwicklung an anderen, für die Phonation bedeutungslosen Stellen beginnt, dieses Frühsymptom völlig fehlen kann und auch wirklich fehlt, sodaß demnach nichts vorhanden ist, was den Patienten auf die Entstehung einer derartigen Erkrankung hinweist. Da Schmerzen oder Beschwerden anderer Art kaum vorhanden sind, von Vielen gewisse unbestimmte Empfindungen in der Tiefe des Halses, welche zeitweilig vorhanden sein können, nicht weiter beach tet werden, so ist demnach, abgesehen von der Heiserkeit bei dem bestimmten Sitze am Stimmbande, das Fehlen der Symptome das Gewöhnliche. In diesem Falle gelangen die Fälle auch immerhin erst später zur Beobachtung, wenn mit zunehmendem Wachstum sich Beschwerden allmählich entwickeln.

Bei der Bevorzugung, welche die Stimmbänder selbst beim Carcinom erfahren, ist es die Heiserkeit und namentlich die allmählich und langsam zunehmende, welche den Kranken veranlaßt, ärztliche Hilfe aufzusuchen. Obwohl diesem Symptom, welches nur einem mechanischen Hindernis der Stimmbildung seine Entstehung verdankt, keine für den Krebs pathognomonische Bedeutung beizulegen ist, so ist es doch in vielen Fällen das einzige frühzeitige, welc:les Beachtung verdient und dessen Entstehungsursache durch die Kehlkopfspiegeluntersuchung von seiten des untersuchenden Arztes in dem konkreten Falle aufgeklärt werden kann.

Wir besprechen zunächst die an den Stimmbändern auftretenden Carcinome und berücksichtigen später die anderen Lokalisationen, soweit sich hierbei wesentliche Varianten ergeben. An den Stimmbändern beobachten wir am häufigsten das Carcinom in seiner Entstehung und Frühform unter dem Bilde einer zirkumskripten, kleineren Geschwulstbildung, wie eines Polypen, jene Form, für welche auch B. Fraenkel den Namen Carcinoma polypoides im klinischen Sinne gewählt hat. Es stellt sich diese Bildung dar im Beginne als eine flachere oder etwas breiter aufsitzende Prominenz von rundlicher, kugeliger oder länglicher Gestalt mit glatter oder meist etwas höckeriger oberfläche von verschiedener Farbe. Letztere kann rötlich, rötlich-weiß sein oder etwas undurchsichtig Opakes an sich tragen und durch diese letztere Eigentümlichkeit schon eine gewisse Fremdartigkeit, welche nicht zur Schleimhaut gehört, dokumentieren. Dieser bisher noch kleine und unscheinbare Tumor zeigt schon, wenn man sorgfältig darauf achtet, einen etwas tieferen Sitz in der Schleimhaut. einen innigeren Zusammenhang mit derselben, als ob er aus der Tiefe des Gewebes hervorsprießt; er läßt sich vielfach nicht scharf abgrenzen gegen die Nachbarschaft, wie wir dies sonst bei gutartigen Tumoren zu beobachten gewöhnt sind. Allerdings liegen ganz vereinzelte $\mathrm{Be}$ obachtungen über gestielte Larynxcarcinome vor; indes dürften diese schon nach der Spärlichkeit der Mitteilungen zu den höchst seltenen Ausnahmen gehören, welche der gewöhnlichen Darstellung entfallen. Zu den angeführten Momenten kommt noch hinzu das Aussehen und die Farbe des Tumors, obschon, wie wir sogleich sehen werden, hier eine besondere Vorsicht am Platze ist. Die Farbe des am Stimmbande auftretenden Carcinoms wird verschieden angegeben; sie kann rötlich, weißrötlich, weifopak sein; letztere Farbe, das weißkreidige opake Aussehen dieser Tumoren, hat, wie bereits bemerkt, B. Fraenkel als in vielen Fällen besonders charakteristisch beschrieben. Im allgemeinen ist nicht zu leugnen, daß bei manchen kleinen Carcinomen in der Tat diese Farbennuance zu beobachten ist, aber sie ist nicht regelmäßig vorhanden und überdies nicht nur beim Carcinom; sie ist auch bei andern Tumoren, den sogenannten Papillomen, den papillären Fibromen und gewissen pachydermischen Verdickungen nicht malignen Charakters zu sehen, sodaß demnach die Bewertung der Farbe des Tumors für die Diagnose erheblich reduziert wird. Dasselbe gilt von jener entzündlichen Infiltration in der Umgebung der Geschwulst, welche Jurascz angeblich bei Larynxcarcinomen beobachtet hat und welche gerade als pathognomonisch für Carcinom von ihm erklärt wurde. Alle Laryngologen sind einmütig der Ansicht. daB eine entzündliche Infiltration in dem benachbarten Gewebe dem Carcinom nicht eigentümlich ist und demgemäß derartige Rötungen und Schwellungen für das Carcinom absolut nicht pathogonomisch sind. Auch die Konsistenz des Tumors, auf welche von mancher Seite für die Feststellung der Diagnose Wert gelegt wird, hat keine pathognomonische Bedeutung, da ja diese je nach der Beschaffenheit des Gewebes, nach dem Blutgehalt etc. sowohl bei bösartigen Tumoren wechseln, anderseits bei gutartigen Gebilden die gleiche sein kann wie bei malignen.

Durch die angeführten Momente, Alter, Geschlecht, Entstehung und Aussehen des Tumors etc., wird beim untersuchenden Arzte der Verdacht eines beginnenden Carcinoms des Larynx schon frühzeitig erweckt, in einigen Fällen wird die Diagnose eines solchen bereits zur Wahrscheinlichkeit, aber ihre Sicherheit wird dadurch nicht gewährleistet. Hier muß mit der Erfahrung gerechnet werden, daß es eine ganze Reihe anderer Prozesse gibt, welche ein dem beschriebenen höchst ähnliches, wenn nicht annähernd gleiches Bild bieten. In dieser Hinsicht entscheidet das Alter und Geschlecht nicht, da die in Frage stehenden Affektionen keine Auslese treffen. Es können in Betracht kommen entzündliche, zirkumskripte lnfiltrationen gutartiger Natur oder Infiltrationen, wie wir sie im Verlaufe früherer Infektionen (Lues, Tuberculose, Lupus, Lepra etc.) vielfach im Larynx $\mathrm{zu}$ beobachten pflegen. Weiterhin sind hervorzuheben die gutartigen Tumoren, Fibrome etc., welche unter Umständen sich nicht in einem ganz eindeutigen Gewande präsentieren. Hält man noch damit zusammen, daf es sogenannte Mischformen von gutartigen und malignen Gewächsen gibt, wie wir Krebse in papillären Geschwülsten sich entwickeln sehen, so ergeben sich hieraus die Schwierigkeiten der Diagnose sehr leicht. Zur Beseitigung der etwaigen Zweifel werden wir, wie ich dies später ausführen werde, zu einem weiteren Hilfsmittel unsere Zuflucht nehmen müssen, zur mikroskopischen Untersuchung exstirpierter Stückchen.

Die zweite Form, unter der sich der beginnende Kehlkopfkrebs dem Beobachter darstellt, ist die flächenhaft entstehende Infiltration, wobei also nicht ein kleinerer oder größerer zirkumskripter Tumor sichtbar ist, sondern eine sich über eine größere Partie der Schleimhaut hinziehende Schwellung. Auch hier ist bevorzugt das wahre Stimmband, obschon auch andere Teile des Larynx nicht verschont bleiben. Die betreffende erkrankte Partie des Kehlkopfes zeigt sich bei der laryngoskopischen Untersuchung in Form einer diffuseren Schwellung von verschiedener Färbung, grau, graurot bis zur intensiveren Rötung. Sitzt der Prozeß am wahren Stimmband, so erscheint dessen Form hochgradig verändert; seine dreiseitig prismatische Form ist umgewandelt in eine Art Walzenform; der scharfe Rand der Stimmlippe ist verschwunden, die Oberfläche des Stimmbandes ist nicht mehr glatt, sondern höckerig granuliert.

Da bei dieser Form der Erkrankung der Charakter einer Geschwulstbildung von Hause aus schon nicht ausgesprochen ist, so ist sie im Beginne als carcinomatöse schwer zu erkennen. Andere entzündliche Prozesse, Schwellungen der Schleimhaut bei Potatoren, Erkrankungen des Larynx im Verlaufe einer inveterierten Syphilis, Infiltrationen tuberculöser Natur bieten dasselbe Bild. Und ganz besonders erwähnenswert ist die Pachydermia laryngis, welche zu diagnostischen Irrtümern Veranlassung geben kann. Es kommt bei dieser Erkrankung, wie dies Virchow zuerst beschrieben hat, am hinteren Ende der Stimmbänder zu Wulstbildungen, in deren Mitte sich eine grubenartige Vertiefung bildet, welche pathologisch-anatomisch als epitheliale Wucherungen mit Verdickung des epidermoidal gewordenen Epithels und zahlreichen, dicht nebeneinander stehenden Papillen unter dem Epithel sich darstellen. Diese Papillen, zwar meist nur mikroskopisch sichtbar, erscheinen in seltenen Fällen auch makroskopisch als dicke, faltenartige Auswüchse mit epidermoidalem Ueberzuge, sodaß auch bei der eigenartig opaken Farbe, welche diese Verdickungen erkennen lassen, eine gewisse Aehnlichkeit mit dem Carcinom naheliegt und besonders dann noch mehr, wenn Einrisse und Furchenbildungen, wie man sie auch zeitweise am Carcinom sieht, makroskopisch sichtbar sind. 
Zur Beseitigung der diagnostischen Zweifel sind nun viele Momente herangezogen worden. In erster Linie wird auf die Einseitigkeit der Erkrankung beim Carcinom hingewiesen. gegenüber der doppelseitigen Erkrankung aus anderen Ursachen, beispielsweise bei Syphilis. Die Erfahrung spricht nicht zugunsten dieser Ausnahme, da auch im Verlaufe der Syphilis einseitige Erkrankungen vorkommen können, ebenso wie bei den tuberculösen Infiltrationen des Larynx, welche bekanntlich ein- und doppelseitig, vielleicht noch häufiger einseitig auftreten. Ueberzeugt man sich weiterhin, dab das Alter und das Geschlecht der Erkrankten für die Beurteilung des krankhaften laryngealen Prozesses auch jede Bedeutung verlieren, da ja Lues. Tuberculose und Pachrydermie in dieser Hinsicht keine Ausnahme machen. so sieht man die diagnostischen Schwierigkeiten nur zunehmen. F. Semon hat als frühzeitiges Symptom des beginnenden Larynxkrebses die verminderte Bewegungsfähigkeit des carcinomatösen Stimmbandes angegeben, welche wegen der in die Tiefe gehenden Infiltration schon frühzeitig gehemmt sein sollte.

Diese Beobachtung hat eine Bestätigung nicht erfahren; eine etwaige Bewegungsstörung wird zunächst nicht in allen Fällen von Stimmbandcarcinom beobachtet, und überdies kommt sie auch zustande bei allen anderen pathologischen Prozessen. welche vermöge ihres Sitzes und ihrer Ausdehnung Bewegungsstörungen auf rein mechanischem Wege erzeugen können, ganz besonders, wenn in der Nähe der Cricoarytaenoidealgelenke oder an diesen selbst Schwellungen entzündlicher Natur auftreten. gleichgültig. welcher Art sie sind, ob gutartig oder bösartig. sodaß also beim Vorhandensein dieser Störung auf die Pathogenität des Prozesses Rückschlüsse nicht gestattet sind.

Wir haben bisher die an den Stimmbändern auftretenden zwei Hauptformen des Larynxkrebses in ihrer Frühform betrachtet. Aehnliche Bilder bieten auch diejenigen Carcinome dar, welche an anderen Stellen des Kehlkopfes beginnen, so an den falschen Stimmbändern, an der Epiglottis und im Ventriculus Morgagni. Auch hier kann man sie entstehen sehen in zirkumskripter und diffuser Form, und sie sind bei der laryngoskopischen Lntersuchung von gutartigen Tumoren oder entzündlichen Infiltrationen zunächst kaum unterscheidbar. Der Verdacht eines Carcinoms wird erregt, wenn man den tiefen Sitz des Herdes in der Schleimhaut, seine geringe Abgrenzung von dem unterliegenden Gewebe feststellen kann. Die Farbe ist meist diejenige der Schleimhaut, manchmal ein etwas gesättigteres Rot, sodab jenes opake Grau, welches dem Carcinom an den Stimmbändern zeitweilig anhaftet. hier nicht sichtbar ist. Auch die Konsistenz bietet für die Diagnose keine Anhaltspunkte.

Am leichtesten erkennbar ist das Carcinom an der Epiglottis, welches vielfach seine ersten Erscheinungen am freien Rande zeigt, da infolge der relativen Dünnheit der hier befindlichen Schleimhaut bei der Nähe des Knorpels der mehr oder weniger oberfächliche Sitz des Tumors und sein Verhalten zur Nachbarschaft leicht zu übersehen ist.

Das aus dem Ventriculus Morgagni hervor wuchernde Carcinom, wie ein solches von F. Semon beschrieben worden ist, weiterhin das von den Schleimhautwänden des Ventriculus ausgehende (Carcinoma ventriculare B. Fraenkels) und endlich das von M. Schmidt noch besonders erwähnte, in der Tiefe des Larynx sich entwickelnde Carcinom bieten für die Diagnose besondere Schwierigkeiten, da besonders bei den letzteren beiden Formen infolge der tiefen Lage zunächst nur durch Auftreibung der Nachbarschaft und Vordrängung der Gewebe ihr äuferes Relief sich bemerkbar macht. Hier kann neben der sichtbaren Schwellung und etwaiger ödematöser Infiltration schon frühzeitig jene von Semon angegebene Bewegungsstörung des betreffenden Stimm. bandes die Aufmerksamkeit des Untersuchers fesseln und auf diese Weise zur besonderen Beachtung der weiteren Symptome Veranlassung geben. Namentlich sind Papillombildungen, welche an der Oberfläche sich zeigen und nach der operativen Beseitigung stets wiederkehren, verdächtig. Es kann dies eintreten infolge des Roizes des in der Tiefe sitzenden, aber noch nicht sichtbaren Carcinoms, welches nach einiger
Zeit zwischen den papillären Excrescenzen als Knötchen erscheint und sich $\mathrm{zu}$ weiterem Wachstum anschickt. Die Sicherheit der Diagnose ist indes in Anbetracht etwaiger Verwechslungen mit anderen pathologischen, nichtkirebsigen Affektionen nicht gegeben. Namentlich können tuberculöse, lupöse und syphilitische Erkrankungen ein ähnliches oder scheinbar fast gleiches Krankheitsbild darbieten, sodal die Differenzierung mittels des Kehlkopfspiegels unmöglich erscheint. Um hier die Fehlerquellen auszuscheiden, ist es wichtig, den gesamten äußeren Habitus der Kranken zu berücksichtigen, den Lungenbefund festzustellen und namentlich den etwaigen Auswurf auf Tuberkelbacillen zu untersuchen. Der positive Befund wird zur Klärung der Diagnose beitragen, der negative aber nicht unbedingt gegen die Annahme eines Carcinoms verwendet werden können; überdies ist auch die Möglichkeit des Vorkommens von Mischformen von Tuberculose der Lungen und Carcinom des Larynx festzuhalten. Das Gleiche gilt für etwaige syphilitische Prozesse, welche sich im Larynx etablieren können, entweder isoliert oder mit Carcinom zusammen. Hier müssen für die Diagnosenstellung herangezogen werden die Anamnese, die Untersuchung des übrigen Körpers auf Narben und andere verdächtige Momente und endlich die Anwendung antisyphilitischer Mittel, sei es großer Gaben Jodkalium oder einer Inunktionskur. Die syphilitischen Prozesse pflegen unter einer derartigen Therapie sich zur Abheilung anzuschicken, während die Carcinome, auch wenn sie sich selbst durch Jodkalium etwas verkleinern lassen, was zeitweilig beobachtet wird, niemals zum Schwinden gebracht werden, sondern im Gegenteil ein weiteres Wachstum zeigen.

Ueber die Möglichkeit der Verwechslung des Car. cinoms mit gutartigen Tumoren, Fibromen etc., mit der Pachydermie habe ich bereits früher gesprochen. Sind demnach trotz Abwägung aller Momente und Möglichkeiten, wie aus den Auseinandersetzungen hervorgeht, die Schwierigkeiten der frühzeitigen Erkennung des Kehlkopfkrebses recht grofe, so müssen wir nach weiteren Möglichkeiten, sie zu beseitigen, Umschau halten. Und in dieser Hinsicht bleibt als wichtigstes Hilfsmittel die mikroskopische Untersuchung exstirpierter Stückchen der Geschwulst, bzw. der krankhaften Neubildung. Aber auch diese Methode hat ihre bestimmte Begrenzung. Einmal ist sie nicht stets anwendbar, da man nicht in allen Fällen auf endolaryngealem Wege Stückchen exstirpieren kann, namentlich schon nicht bei ganz flachen Neubildungen, und weiterhin ist das Resultat nur dann beweiskräftig, wenn der Nachweis eines Carcinoms sicher gelingt; der negative Befund fördert unsere Diagnose nicht, da er nicht gegen die Anwesenheit eines Carcinoms spricht. Nur unter dieser Voraussetzung kann die probatorische Exstirpation diagnostisch verwertet werden. Es soll ein möglichst grobes Stück und womöglich aus der Tiefe entnommen werden. Deshalb eignet sich das von Schrötter angegebene, harpunenartige Instrument für diese Zwecke nicht besonders, vielmehr sind scharfe, schneidende Doppelkuretten zu empfehlen, mit welchen, nach vorheriger, lokaler Anästhesie mit Cocain-Adrenalin, Probestücke entfernt werden können. Dab die mikroskopische Untersuchung von fachmännischer Seite ausgeführt oder kontrolliert werden soll, ist eigentlich selbstverständlich, damit etwaige Irrtümer, welche unterlaufen können, so weit als möglich ausgeschlossen werden. Durch eine sorgfältige Untersuchung derartig und besonders aus der Tiefe exstirpierter Partien, möglichst in Serienschnitten, gelingt in den meisten Fällen die Sicherstellung der Diagnose in den Frühformen des Carcinoms. Es ist aber festzuhalten, dab auch bei vorhandenem Carcinom der mikroskopische Befund ein negativer sein kann und auch jedesmal sein mub, wenn bei der Exstirpation das carcinomatöse Gewebe nicht mitgefaßt wird, was durchaus nicht zu den Seltenheiten gehört. Es geschieht dies meist bei sehr tiefem Sitze des Carcinoms oder besonders, wenn oberhalb desselben pachydermische Verdickungen auftreten oder entzündliche Infiltrationen irgend welcher Art, oder wenn Komplikationen von tuberculösen oder syphilitischen und anderen Prozessen mit Carcinom vorhanden sind, wie solche Fälle durchaus nicht gerade zu den Seltenheiten zu gehören pflegen.

Diese Erfahrungen mahnen zu grober Vorsicht und er- 
fordern im Fallo eines negativen Befundes die wiederholte Untersuchung exstirpierter Stücke, sei es, daß die Exstirpation auf endolaryngealem Wege ausgeführt wird, oder in zweifelhaften Fällen nach vorheriger Spaltung des Kehlkopfes, wenn die Schwierigkeit der endolaryngealen operation aus irgend einem Grunde nicht überwunden werden kann. (SchluB folgt.) 